\title{
Hand-held Optical Fiber Smartphone Spectrometer for Classification of Vegetable Oils
}

\author{
Md Arafat Hossain ${ }^{1,2}$, John Canning ${ }^{1, *},{\text { Kevin } \text { Cook }^{1} \text { and Abbas Jamalipour }}^{2}$ \\ ${ }^{1}$ interdisciplinary Photonics Laboratories (iPL), School of Chemistry, The University of Sydney, NSW 2006, Australia \\ ${ }^{2}$ School of Electrical and Information Engineering, The University of Sydney, NSW 2006, Australia \\ Email: john.canning@sydney.edu.au
}

\begin{abstract}
An optical-fiber-based low-cost, hand-held smartphone spectrometer is demonstrated for differentiating vegetable oils. The visible fluorescence spectrum of extra virgin olive oil is found to be significantly different from other oils, thus making forensic identification straightforward.

OCIS codes: (300.8280) Spectroscopy, fluorescence and luminescence; (280.4788) Optical sensing and sensors; (999.9999) Optical-fiber spectrometer; (999.9999) Smartphone spectrometer; (999.9999) Internet of Things
\end{abstract}

\section{Introduction}

There are now a number of micro spectrometers that address the agricultural and food sectors [1] indicating the importance of spectroscopy within these sectors. Our recently reported smartphone spectrometer offers an alternative, allowing wireless connectivity, Internet of Things (IoT) compatibility, green power accessibility and efficiency, and more. We have demonstrated lab-in-a-phone technology by making suitable hand-held devices for ubiquitous sensing of chemical environments [2-5]. Multiple spectroscopic functionality, for example, is demonstrated using the smartphone's high sensitive CMOS detector with a low-cost dispersive element [3]. Data can be shared or processed remotely via the internet, perhaps through a digitized cloud collecting data from many of such instruments or combined to allow real-time mapping within a potential IoT application [4]. The use of an optical fiber based bundle extends the spectrometer's capability further by collecting light from difficult to access places as well as avoiding unwanted stray light illumination [5]. Herein, we have advanced our previously reported optical fiber smartphone spectrometer by integrating an array of ultra-violet (UV) LEDs for generating fluorescence with the endoscope using a 3D printed support. Visible fluorescence spectroscopy with this instrument is applied to identify the type of vegetable oils. Specifically, we show that fluorescence spectroscopy can identify the visible emission bands of oxidation components within the oils - these fluoresce and are responsible for the degradation of essential chemical compounds within the oil such as unsaturated fatty acid [6]. The device demonstrates significant potential in rapid, on-site identification of food quality, able to engage consumers with quality control, more broadly opening up the quality assurance market to the wider public aided by IoT connectivity. This assurance is crucial for the expansion of Australian food into the region given they are subject to growing criminal imitations and fraud, damaging the internationally recognised Australian brand.

\section{Spectrometer design and fabrication}

The 3D design of the optical fiber spectrometer is shown in Fig. 1. A flexible fiber bundle probe (Edmund Optics, FiberScope, $L=60 \mathrm{~cm}$ ) integrated onto a designer smartphone spectrometer platform is used to capture fluorescence emission therefore removing ambient illumination. In order to generate fluorescence, three UV LEDs $\left(\lambda_{\mathrm{ex}} \sim 370 \mathrm{~nm}\right)$ are placed on the periphery of a circular end cap that holds the end of the fiber bundle probe at the centre. These parallel connected LEDs are separated equally to each other so that they produce uniform illumination to the sample surface and powered by the smartphone's micro USB port via an "On the Go" (OTG) cable which offers voltage up to $V \sim 5$ volts and a maximum current of $I \sim 500 \mathrm{~mA}$. A resistor $(R=12 \Omega)$ is used to limit $I$ and $V$ to the LEDs. The fluorescence emission is collected through the collection fiber bundle, positioned at the centre of the fiber bundle

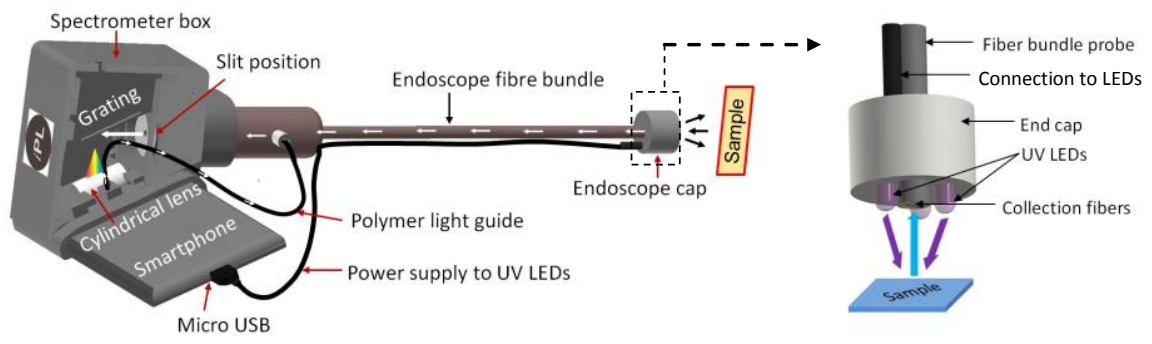

Fig. 1. 3D CAD design of the smartphone optical fiber spectrometer. 
probe where an objective lens at the probe end increases light collection. The collected light is then collimated using the second lens on the other end of the probe and subsequently diffracted by a low cost dispersive element (DVD surface, $A=3 \times 1.5 \mathrm{~cm}^{2}$ ). This diffracted light is finally imaged and calibrated ( $I$ vs $\lambda$ ) across the CMOS chip (details in [5]). A cylindrical lens is also used to improve light collection per pixel on the detector. The 3D CAD design of the spectrometer box is optimized for a Samsung Galaxy S4 smartphone and printed using a 3D printer. An Android app is also designed to perform the spectral measurements and share the results with other devices.

\section{Spectra measurements of vegetable oils}

The optical fiber smartphone spectrometer measured the fluorescence spectra of some commonly used vegetable oils. The sample oils used were collected from the local supermarket (Fig. 2a). From visual inspection, the variation in color shades has no obvious correlation between oil types making it hard to distinguish the various vegetable oils. For example, determining normal olive oil and extra virgin olive (EVO) oil is not obviously feasible - given the ease with which consumers can be charged more for the extra virgin oil, a simple means of differentiating the two quickly and on the spot to ascertain quality assurance would be ideal. The fiber bundle probe is butted against the surface of the oil's container. After measuring the fluorescence spectra, shown in Fig. 2b, a significant decrease of the blue bands $(\lambda \sim 452 \mathrm{~nm})$ and increase in the red bands $(\lambda \sim 600 \mathrm{~nm})$ are observed in EVO oils than all other oils. Identical fluorescence responses are also recorded among different brands of EVO oils (EVO 1 and 2 are respectively for ollo and red island brands). The anti-oxidant vitamin $E$ band at $\lambda \sim 525 \mathrm{~nm}$ is found stronger in EVO oils than the other refined vegetable oils. From the spectral analysis, a coefficient $k$ is obtained (Fig. 2c) by normalizing the vitamin $E$ band $(\lambda \sim 525 \mathrm{~nm})$ with the oxidizing products emission band $(\lambda \sim 452 \mathrm{~nm})$. A 4 -fold increase of the $k$ value is recorded in EVO oils than that of the other vegetable oils. For the extra virgin olive oils, the relative intensity of the oxidation product band is found to be very weak compared to the other oils. This is because olive oils are generally manufactured by cold mechanical pressing to preserve their anti-oxidants.
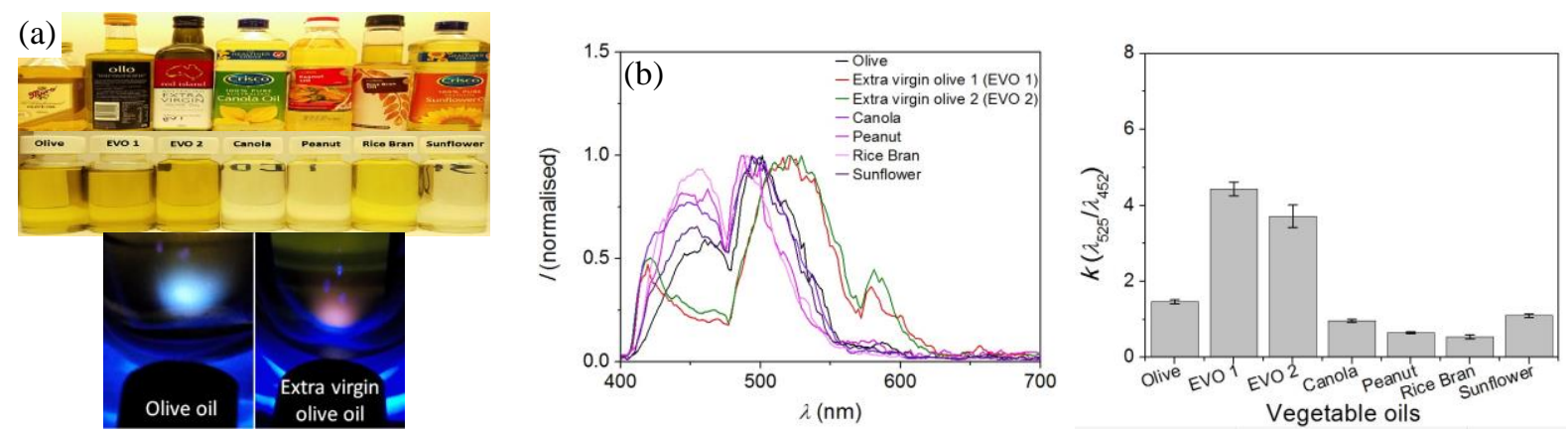

Fig. 2. (a) Sample vegetable oils collected from a supermarket and fluorescent imaged of extra virgin olive and normal olive oils; (b) fluorescence spectra of the vegetable oils measured on the smartphone spectrometer and (c) plot of $k$ for different oils.

\section{Conclusion}

A hand-held smartphone spectrometer with an optical fibre endoscope probe and UV LEDs encased in a custom 3D printed support, allows fluorescence signal collection from difficult to access places such as inside of a shipping tank. The results show strong fluorescence bands characteristic of strong oxidizing components present within the more refined oils compared to the extra virgin vegetable oils. These components are unwanted and generally regarded detrimental to health. In conclusion, the work supports the concept of rapid field diagnostics during processing, storing and marketing, making interactive customer service and assurance one step closer to fruition.

\section{References}

[1] A.G. Mignani et al, "Eat-by-Light: Fiber-optic and micro-optic devices for food quality and safety assessment," IEEE Sensors J. 8, 1342-1354 (2008).

[2] M. A. Hossain, J. Canning, S. Ast, P. J. Rutledge, T. L. Yen, and A. Jamalipour, "Lab-in-a-phone: smartphone-based portable fluorometer for pH measurements of environmental water,” IEEE Sens. J. 15, 5096-5102 (2015).

[3] M. A. Hossain, J. Canning, S. Ast, K. Cook, P. J. Rutledge, and A. Jamalipour, "Combined "dual" absorption and fluorescence smartphone spectrometers," Opt. Lett. 40, 1737-1740 (2015).

[4] M. A. Hossain, J. Canning, S. Ast, P. J. Rutledge, and A. Jamalipour, "Early warning smartphone diagnostics for water security and analysis using real-time pH mapping," Phot. Sensors 5, 289 (2015).

[5] M. A. Hossain, J. Canning, K. Cook, and A. Jamalipour, "Optical fiber smartphone spectrometer," Opt. Lett. (in early post paper February 2016).

[6] Y. G. M. Kongbonga, H. Ghalila, M. B. Onana, Y. Majdi, Z. B. Lakhdar, H. Mezlini, S. S. Ghalila, "Characterization of vegetable oils by fluorescence spectroscopy" Food and Nutr. Sci. 2, 692-699 (2011). 